\title{
Una scrittura in maschera
}

\author{
Giulia Calligaro
}

\begin{abstract}
Nella prima parte del suo saggio l'autore riflette sugli impieghi del dialetto nel teatro italiano contemporaneo, mettendone in luce gli aspetti non naturalistici, ma espressivi, e constatando l'abbondanza degli esperimenti linguistici del teatro meridionale. Si sofferma, poi, sul caso di Enzo Moscato, caposcuola della nuova avanguardia napoletana, di cui analizza l'opera Signurì Signurì. Di essa si rilevano con puntualità sia i modi pluringustici di una scrittura barocca che mescola registri, stili e idiomi, sia la ossessività dei temi funerei e sessuali. L'insieme dell'opera dell'autore viene letta alla luce di categorie junghiane.
\end{abstract}

Parole chiave: teatro contemporaneo, dialetto, Moscato.

\begin{abstract}
In the first part of his paper, the author reflects on the exploitation of dialect in contemporary Italian theatre, highlighting the non naturalistic yet expressive aspects as well as exploring the abundance of linguistic experiments in Southern theatre. It goes on to focus on Enzo Moscazo, a front runner in the new Neapolitan avant-guard, analysing his work entitled Signurì Signurì. From this work both the poly-linguistic methods of Baroque writing that mixes registers, styles and languages, and the obsession with funereal and sexual themes become obvious. The entire works of the author are read in the light of Jungian concepts.
\end{abstract}

Key words: Contemporary theatre, dialect, Moscato.

Mentre l'alchimia, grazie ai suoi simboli, è come il Doppio spirituale di un'operazione che risulta efficace soltanto sul piano della materia reale, il teatro deve essere a sua volta considerato il Doppio, non di quella realtà quotidiana e diretta di cui è a poco a poco divenuto soltanto la copia inerte, vana quanto edulcorata, ma di un'altra realtà rischiosa e tipica, dove i principî, come i delfini, una volta mostrata la testa, $s^{\prime}$ affrettano a reimmergersi nell'oscurità delle acque. 


\section{Dialetto, lingua speciale del teatro}

Non sorprende certo che l'Italia, paese dalla discontinua tradizione teatrale in lingua, vanti una solida traccia dialettale, resa fertile proprio dalla particolare situazione linguistica nazionale. Bastino ad esempio i nomi di Ruzante, di Carlo Maria Maggi, di Carlo Goldoni e prima di lui si richiami alla mente la tradizione plurilinguistica della commedia dell'arte. Ma se fino ad Eduardo De Filippo i grandi commediografi in dialetto sorgevano in regioni di ampia dialettofonia, dove la lingua minore era condivisa con una vasta comunità di parlanti, oggi questo tipo di teatro, oltre a riprendere le tradizioni maggiori, utilizza varianti periferiche delle parlate locali, spesso in commistione con linguaggi alti e tecnici, nonché con riferimenti colti e letterari; il tutto, si direbbe, più in funzione espressiva che mimetica. ${ }^{1}$ Avanza qui l'ipotesi che sia avvenuto nel teatro qualcosa di simile a quanto è successo nella poesia neodialettale italiana del secondo Novecento: ovvero che la scelta della lingua minore sia fatta proprio in funzione della sua marginalità e in risposta alla diminuita forza evocativa della lingua maggiore. ${ }^{2}$ Di più: che lavori al fondo un ritorno all'originario teatro del significante ${ }^{3}$ che fa della lingua la propria protagonista, pur oggi con finalità nuove rispetto al passato. Sia, perciò, questa lingua, più figlia di Viviani e di Testori che di Eduardo, e infine, che si tratti di una lingua speciale del teatro, restando il dialetto una delle soluzioni più teatrali tra quelle offerte dal panorama nazionale. Fatto, questo, probabilmente dovuto alla possibilità di rinvenire entro queste aree espressive una forte potenzialità simbolica, capace di riferirsi ad una mitologia condivisa, cosa indispensabile per una creazione che non sia semplice uso del teatro come luogo di racconto di storie; nonché al suo costituire un genere veramente radicato nella natura e nella lingua teatrale italiana, avvicinando un'operazione profondamente letteraria e una, altrettanto profonda, di controcanto (pur sempre letterario) alla letteratura canonica, in cui la prima è fecondata teatralmente dalla seconda, riconducibile alla tradizione della commedia, cui il teatro italiano è stato in passato più vocato: con la novità che ora il dialetto ha assunto su di sé anche responsabilità tragiche.

Detto questo, va chiarito che potenzialità e quantità non vanno insieme, e infatti, accanto a quella dialettale, è in grande voga oggi in Italia una drammaturgia che potremmo definire minimalista o generazionale, di ascendenza anglossassone, che porta in scena storie rasoterra in un parlato sporco, in genere giovanile, di forte impronta cinematografica, che però, a differenza di quanto avviene nella terra originaria, qui appare epigona, proponendo un vissuto che non trova sostegno nella realtà: con il sospetto, dunque, che si tratti più di una moda che di una reale espressione del teatro nostrano. Tant'è che, rag-

1. Cfr. Puppa, Il nuovo teatro italiano, in: E. CeCCHI e N. Sopegno (a cura di), Il Novecento, Milano: Garzanti, 2003, p. 178-195.

2. Cfr. Pier Vincenzo Mengaldo, La tradizione del Novecento, Quarta serie, Torino: Bollati Borlinghieri, 2000, p. 8-11.

3. Le definizioni "comico di significante»/ «comico di significato» si devono a Maria Luisa Altieri Biagi, La lingua in scena, Bologna: Zanichelli, 1980. 
giunta in questa scrittura una trasposizione quasi totale del parlato reale, immediatamente la mimesi cede a nuovi fenomeni di eversione linguistica (letterariamente guidati in direzione substandard), a confermare come la natura intima della drammaturgia italiana non sia probabilmente quella volta al naturalismo del dettato. ${ }^{4}$

Ritornando alla scrittura dialettale per il teatro e passando ad una rapida panoramica contemporanea, si possono individuare vari repertori regionali: quello romagnolo rappresentato da Raffaello Baldini, teatrale anche quando scrive versi; quello toscano che fa capo a Ugo Chiti, quello piemontese che dirama dalla scuola del Teatro Settimo di Gabriele Vacis e Laura Curino, incrociandosi, via "teatro di narrazione», anche con il filone veneto di Marco Paolini (ma per il Veneto va ricordato almeno anche il pavano di Giuliano Scabia); quello ancora tra Piemonte e Lombardia, ma riformulato in un personale idioletto, di Antonio Tarantino, e quello di area triestino-mitteleuropea rappresentato dalla "dialettalità sotterranea» del germanista Claudio Magris.

E però il sud d'Italia a rappresentare il vero caso del teatro dialettale, mescolando nella scelta linguistica un intento di sottolineata marginalità sociale eppure di grande consapevolezza letteraria. Un nutrito filone drammaturgico si annida nella Sicilia di Franco Scaldati e nel suo «maledettismo sociale», del messinese Spiro Scimone che propone duetti, interpretati da lui e da Francesco Sfarmeli, a mezzo tra evocazione del passato e desolazione del presente, e del più giovane Davide Enia. E anche se questi ultimi parrebbero alludere ancora ad aree di ampia condivisione dialettale, in realtà si assestano su scelte minoritarie all'interno della variante maggiore, più prossime al conio di un idioletto che al rimando diretto ad una tradizione. A conferma di ciò si noti come emergano oggi da queste geografie sociologicamente affini anche lingue non tradizionalmente teatrali, quali il calabrese di Francesco Suriano.

La capitale di questo teatro resta comunque Napoli, che vanta anche in epoca moderna la quota di maggioranza nella nuova scrittura teatrale: è quella di Ruggero Cappuccio e dei suoi accostamenti anglopartenopei (ad esempio in Shakespeare di Napoli), o di Annibale Ruccello, che propone cortocircuiti linguistici ed emotivi tra italiano e dialetto, e, naturalmente, di Enzo Moscato, di cui si parlerà più diffusamente.

Sia precisato ancora una volta, però, che i drammi dei nuovi drammaturghi napoletani non sono solo il frutto della tradizione consolidata in quella

4. Capofila di questa linea drammaturgica è Fausto Paravidino (Genova 1976), autore giunto alla fama giovanissimo con testi che fanno chiaro riferimento all'inglese new angry generation e con i quali viene portata in scena la vita dei giovani venti-trentenni di oggi (Trinciapollo, Gabriele, Due Fratelli - Premio Tondelli 1999, e Premio Ubu novità italiana 2001 - Tutta Colpa di Cupido, La Malattia della Famiglia M, Natura Morta in un Fosso, Genova 01 e Noccioline - Nuts, in gran parte raccolti in Fausto PARAVIDINO, Teatro, Milano: Ubulibri, 2002. Ma l'ancor più giovane Letizia Russo (Roma 1980), rifacendosi al medesimo modello, cerca un effetto di realtà attraverso una lingua bassa e stralunata che non è già più naturalistica (il pensiero va, ad esempio, a Tomba di cani, vincitore del Premio Tondelli 2001, categoria giovane del Premio Riccione). 
terra, ma che si ricollegano direttamente a Genet, Pinter, Beckett o Artaud e alla maggiore prosa europea, ovvero che in essi il codice linguistico prescelto non si limita ad essere connotativo di una certa ambientazione, ma è, al contrario, costantemente e strenuamente costitutivo del suo dire, fatto che consente che qualcosa di estremamente circoscritto ad un'area si renda universale. Tutto ciò sarà subito verificato nell'analisi che segue di un testo di Moscato.

\section{Il caso di Enzo Moscato}

«Ha da passa' 'a nuttata», concludeva Eduardo in Napoli milionaria. "Ha da passa' 'a nuttata», ribatte Enzo Moscato (Napoli 1958) in Signurì, signurì che ha debuttato nel febbraio ' 82 a Venezia, in una rassegna organizzata dalla Biennale, salvo che, al termine della citazione, un altro interprete lo freddava con un colpo di pistola, a simboleggiare l'uccisione dei padri della tradizione. ${ }^{5}$ Moscato già dall' 80 era stato consacrato capofila di una nuova avanguardia napoletana con la presentazione di Scannasurice allo Spazio libero di Vittorio Lucariello, la cantina della migliore sperimentazione partenopea. A scandirne il successo sarebbero seguiti poi, per citare solo i testi principali, Ragazze sole con qualche esperienza (1985), Pièce noire (1985), Bordello di mare con città (1987), Rasoi (1991), Mal-d'-Hamlé (1994), Recidiva (1995), Lingua, carne soffio (1996), Teatri del mare (1997): tutti testi in cui Moscato ha dato voce alle contraddizioni della modernità napoletana, depositata senza grazia sulle macerie della tradizione. Non è un caso che il corrispondente retorico che meglio definisce la poetica di Moscato sia, anche a suo dire, l'ossimoro. ${ }^{6}$ Lo si verifica a parti-

5. Le notizie su Enzo Moscato sono tratte da Enrico FIORE, Il rito l'esilio e la peste. Percorsi del nuovo teatro napoletano, Milano: Ubulibri, 2002 e dal saggio di Concetta D'ANGELI, "La lingua cancerosa del teatro, i limiti di Enzo Moscato», pubblicato sulla webzine Ateatro, n. 55, 2003, a sua volta tratto da due tesi di laurea, entrambe discusse presso l'Università di Pisa: la tesi di Martina Rossi, Il travestimento in alcuni testi di Enzo Moscato (anno accademico 2000-2001), e la tesi di Melanie Gliozzi, Enzo Moscato. Per un teatro «sul limite» (anno accademico 2001-2002).

6. Lui stesso in un lungo intervento pubblicato dall'Università di Salerno (www.unisa.it), sito dedicato al teatro napoletano e curato dalla Prof.ssa Antonia Lezza e dal Dott. Gian Paolo Renello, definisce il proprio teatro come un teatro «sul limite», un teatro cioè che si fonda sulla antinomia e sulla dissonanza e che, senza mai tentare minimamente di conciliare i termini oppositivi della contraddizione, al contrario li alimenta, li tiene in vita, e di essi si sostanzia: «Mi è spesso accaduto di praticare drammaturgicamente, sia per iscritto che in scena, questo sconvolgimento d'assetti, questo capovolgimento totale di tratti, quest'ossimoro radicale - paradosso per cui una figura, una cosa, un concetto, è all'unisono, se stessa e il suo esatto contrario - cui il bios stesso della Lingua, in tutta la sua polimorfia, allude». E ancora: «In tutti i miei outcomes scenici, all'esterno come all'interno, macro o micro-scopicamente, a livello di mole o a livello di semplice molecola, tanto per esprimerci (al)chimicamente, ho cercato di evidenziare situazioni oppositive-coincidenti: ordini-disordini, linearità/ingarbugliamenti, sistematicità/eversioni, vale a dire naturali/innaturali tracciati in cui le contraddizioni, gli escludentisi a priori, collidano o si rovescino, aspramente o armonicamente, gli uni negli altri, per tendere a darci un tertium da loro, una essenza/qualità precipua, che, al contempo, conserva e distorce, mantiene e sfigura, le eredità ricevute dalla coppia sottoposta a coniunctio, a innesto, a reazione». 
re proprio dalla lingua, che amalgama frammenti provenienti da sistemi diversissimi. La base è un parlato plebeo e duro, non imborghesito e di struttura arcaica, legato alla sua infanzia di ragazzino povero, trascorsa nei vicoli dei Quartieri Spagnoli di Napoli. Questa base linguistica procede poi verso l'italiano standard, in conseguenza di un'altra fase biografica che lo porta a diventare insegnante e intellettuale. Ma l'italiano non sarà mai l'unica lingua di Moscato: essa coesiste, a pari dignità, con il dialetto e si intreccia con le lingue straniere o antiche, apprese successivamente. Avviene così, nel momento della scrittura, una sorta di processo di diffrazione: sulla lingua d'origine si innestano una terminologia, delle strutture sintattiche, delle espressioni, che hanno provenienze e livelli stilistici molto diversi, insieme a frammenti di lingua letteraria, tratti dalle lingue classiche e dalle lingue straniere moderne (il francese, l'inglese, lo spagnolo, il tedesco) e dal linguaggio patetico e ingenuo della letteratura rosa accanto a sofisticati linguaggi tecnici (ad esempio quello psicoanalitico e quello semiologico), a loro volta mescolati con spezzoni della prosa quotidiana diffusa dalla televisione. Un coagulo abnorme, un «esperanto» linguistico, che trova il più illustre predecessore nel barocco di Giovanni Battista Basile e nel suo Pentamerone, e che dà forma ad una lingua cancerosa, ovvero affetta di quella «peste» di cui Antonin Artaud parlò metaforicamente per esprimere le funzioni più caratterizzanti del teatro. ${ }^{7}$ D'altro canto è lo stesso autore ad annoverare Artaud, insieme a Genet e poi Lacan, Jung e Sade tra i sovversivi cui si ispira la propria idea di teatro. Il risultato è una sorta di «barocco degradato», 8 che "cresce e si gonfia sino al punto di esaurirsi in quanto tessuto verbale significante per avvicinarsi addirittura al grado zero della scrittura [...] di modo che le parole finiscono per confondersi, puramente e semplicemente, con il sordo rumore della vita che passa e si consuma».?

E il modello per il barocchismo di Moscato non può che essere la stessa città di Napoli, ${ }^{10}$ "città soglia», luogo di confine tra la razionalità e una dimen-

7. Ancora Moscato dice: «La mia lingua teatrale — in verità un misto multisonoro e ritmico di napoletano e altri idiomi (italiano, francese, inglese, tedesco, spagnolo, greco, latino, saraceno... ) - un po' è inventata, artificiale, costruita sub vitro come in un'officina alchemica, privata e segretissima, un po' è il ricalco esagerato, iperbolico, ridondante, del caos multietnico-poliglottico che ci gira, ai tempi nostri, attorno. Soprattutto a Napoli, radix-matrix da sempre di strazi-sublimità vocali, d'armonie-cacofonie di echi urbani, decibel, rumori, urla. All'incrocio, dunque, di queste due istanze: rispecchiamento di realtà e superamento della stessa nella pratica fantastica dei suoni, si situa ciò che io parlo a teatro. Che è ovviamente in linea con la tradizione idiomatica dei Padri e in rotta con loro. In linea, perché sempre nella ritualità scritto-scenica dei nostri drammaturghi maggiori (Viviani, De Filippo, Petito, Bracco, Di Giacomo... ) [...]. In altri termini, cerco di esprimere compiutamente quella che, nelle leggi della Retorica, viene definita dimensione ossimorica, cioè la tensione a tenere insieme, senza risolverli in false figure di conciliazione o incastro, tutte le contraddizioni o le opposizioni che una data situazione suole presentarci» (ibidem).

8. E. FIORE, op. cit., p. 74.

9. Ibidem: $74-75$

10. Sempre Moscato: «È così che quando indico Napoli come espressione massima, al contempo concreta e astratta, fecale e sublime, dell'ossimoro, dell'ibrido, della coniunctio oppositorum, non ho timore d'impoverirmi o ridurmi a una mera dimensione local-spettacolare, 
sione misterica e segreta. "È questa antica cultura, che pone al centro l'oltretomba, il mistero e l'irrazionale, quella che, repressa perché sconfitta da una modernità colonizzatrice, riemerge nella forma degradata della superstizione e pervade, spesso innescando altre contraddizioni, tutto il teatro di Moscato». ${ }^{11}$ Trasformando la sua scrittura nel corpo stesso della città, facendola cioè di essa "costitutiva», ${ }^{12}$ come si diceva sopra. Moscato riesce infatti a formalizzare le metastasi dei luoghi comuni che invadono la città senza ricorrere alla forma della denuncia esplicita. Di più: in questo essere corpo da parte della scrittura, esiste una fortissima prossimità tra la lingua, Napoli stessa e il corpo del travestito, personaggio immancabile delle sue pièces. Il travestito è un'immagine di metamorfosi: non possiede infatti una precisa identità, o comunque non accetta un'identità sessuale (quella biologica, cioè quella maschile), ma non ne ha acquisita in modo definitivo una nuova (quella femminile). ${ }^{13}$ Dopo la seconda guerra mondiale, tra le molte cose che a Napoli sono cambiate, anche il ruolo del tradizionale femmeniello si è modificato: il travestito ne ha preso il posto, ma introducendo importanti trasformazioni antropologiche, e di queste prima di tutte lo sradicamento e la solitudine in opposizione alla socialità di vicolo che un tempo si aggregava intorno alla sua figura. "Resta però il senso del confronto con il diverso: anche nella modernità del teatro di Moscato, il travestito rappresenta infatti la possibilità di misurarsi con la parte oscura e profonda della propria individualità e soprattutto interpreta al meglio quella attitudine agli sconfinamenti, che appartengono a Moscato come suo particolare modo di essere, ma che sono anche importanti elementi semantici e formali del suo teatro». ${ }^{14} \mathrm{E}$ inoltre questo personaggio corrisponde effi-

perché la indico come indice ossimorico di ferite universali, coacervo inestricabile di tensionicontraddizioni presenti, passate, future, globali e particolari, generiche e unitissime». L'idea è quella junghiana del misterium coniunctionis (cfr. Gustav Carl JunG, Misterium coniunctionis, in Opere, vol. 14, Torino: Bollati Borlinghieri, 1991). Si tenga conto che dal 1985 Moscato ha iniziato, come documenta Maria Nadotti (cfr. "Enzo Moscato. Di teatro si vive», Linea d'ombra, n. 108, 1995), un'analisi di tipo junghiano, e secondo Jung la tradizione alchimistica e la pratica analitica hanno in comune il tentativo di creare una realtà nuova e superiore: da una parte l'oro, la pietra filosofale, dall'altra la "presa di coscienza» della psicologia moderna. L'alchimia in sostanza è espressione di una pulsione a trasformare la materia prima dell'esperienza in conoscenza. Jung allarga la sua indagine alla saggezza orientale e a esperienze culturali che hanno una radice comune, mostrando come le scoperte scientifiche possano in realtà essere il ritrovamento di universali esperienze.

11. C. D’ANGELI, op. cit.

12. E. FIORE, op. cit., p. 98.

13. «In filigrana il travestito di Moscato rivela le linee del suo modello: quel fermmeniello che, almeno fino alla metà del Novecento, mantiene un importante ruolo sociale nella vita del vico napoletano. Il femmeniello vive di preferenza fra le donne e partecipa strettamente alla loro vita, spesso adornato in modi femminili, con gli orecchini, il trucco, i colori degli abiti delle donne; era considerato indispensabile soprattutto nei giochi di fortuna (il lotto, la tombola), nella persuasione che proprio la sua diversità, il suo essere sulla soglia, né donna né uomo, lo renda il tramite con le potenze metafisiche, con il mondo della magia — che perciò egli abbia un rapporto singolare con la realtà ultraterrena e con la morte» (C. D'ANGELI, $o p$. cit.).

14. Ibidem. 
cacemente al credo filosofico che sostanzia il mondo di Enzo Moscato, ovvero quello della coincidentia oppositorum, elaborato da Carl Gustav Jung nei suoi ultimi studi di psicologia del profondo, in analogia con la fase del processo alchemico nella quale si cerca di visualizzare ed esprimere insieme gli opposti. Mentre «in termini meno filosofici e più teatrali, il travestitismo ha a che fare con l'innaturalismo, che è argomento centrale dell'opera di Moscato». ${ }^{15} \mathrm{E}$, insomma, un amalgama mostruoso di diversità da cui Moscato intende sintetizzare una verità specifica e autentica, riportando così il teatro alle sue origini. ${ }^{16}$

Venendo ora a Pièce noire (Canaria), di cui si tratterà più nello specifico, essa valse a Moscato nel 1985 il prestigioso Premio Riccione (e fu rappresentata nel 1987 per la regia di Chérif, con l'interpretazione di Marisa Fabbri). Il testo incarna appieno la poetica di cui si è tentato di descrivere alcuni tratti. La storia è infatti quella di Ballerina, adesso chiamata "la Signora», che, diventata ricca, spende la vita nel delirante progetto di far materializzare nei propri locali notturni l' "angelo" che mai è riuscita ad incontrare quando faceva la prostituta: prende perciò con sé dei bambini e li cresce educandoli al canto, alla danza, alle lingue antiche, trasformandoli così in "cigni», androgini, eterei e irraggiungibili oggetti del desiderio (Desiderio si chiama, appunto, la sua ultima creazione) e li uccide se acquistano una loro personalità che li porti ad affrancarsi da lei e dal suo progetto.

Cominciamo dunque l'analisi dal titolo. Pièce noire fa diretto riferimento al genere teatrale (pièce) e all'argomento che volge al noir, trattando alla fine anche di un'uccisione. Ma, per un grande studioso di Jung e di alchimia come Moscato, non può essere casuale il riferimento all'Opera al nero, di cui per altro più volte parla. ${ }^{17}$

\section{Ibidem.}

16. Ancora dalle dichiarazioni di Moscato: «Chissà? Forse quel terzo che si raggiunge attraverso l'opera alchemica, l'opera al nero, del fare teatro, è l'Anima, anzi: il fare Anima, come dice James Hillman. E che dunque la messa-in-scena è per l'appunto un rito, una missa sacrale, rivolto al dio di tutte le lacerazioni». E si ricordi che due tra i concetti fondamentali della riflessione junghiana, due funzioni di mediazione tra l'Io e l'inconscio, sono l'animus e l'anima. L'Animus è un aspetto maschile presente nella psiche della donna, l'Anima un aspetto femminile presente nella psiche dell'uomo (cfr. Gustav Carl JunG Studi sull'alchimia, in Opere, vol. 13, Torino: Bollati Borlinghieri, 1997).

17. Nel simbolismo alchemico, l'Opera al nero - una delle quattro della Grande Operaè l'inizio del processo di trasmutazione interiore dell'uomo, cioè il distacco dalle cose terrene. Si chiama così perché consiste in un rinchiudersi in se stessi come in un guscio che, impedendo l'accesso alle sollecitazioni mondane, permette all'uomo di maturare interiormente e di crescere spiritualmente. Il nero è anche il colore della morte, ed è ben scelto perché l'Opera al nero comporta un "morire a se stessi». Non si tratta di una morte fisica, bensì di un morire alle cose del mondo, anche perché l'iniziato deve saper distinguere due modi di morire: l'uno consapevole, e l'altro non consapevole, ed il controllo su se stessi deve essere così raffinato da riconoscere la morte e usarla come veicolo, senza subirla come evento. Il simbolismo alchemico raffigura l'Opera al nero come un guscio che racchiude un corvo, uccello dotato di voce sgradevole all'orecchio. E già da questo simbolismo si può ben capire che, se l'Opera al nero resta fine a sé stessa e non si evolve nei suoi tre stadi suc- 
E si noti che, con precisi riscontri nel processo alchemico, il titolo di Moscato si completa nel «napoletano-scientifico» canaria — specie aviaria delle isole omonime- che nella pièce è rappresentata dagli uccelli tenuti in gabbia dalla Signora (come questa frazione di titolo è racchiusa dalle parentesi) —effigie evidente dei suoi esperimenti umani per formare il «cigno perfetto», l' «angelo»e il cui cicaleccio disturba (!) tutta la storia. Gli stessi uccelli alla fine si riveleranno impagliati, ovvero pura forma. La forma in tutti gli aspetti, come si è detto e come si vedrà, è d'altro canto una delle preoccupazioni maggiori dell'autore.

Passiamo dunque ai personaggi, notando subito come i nomi propri siano perlopiù sostituiti da nomi emblematici o soprannomi-etichetta: la Signora, il suo prediletto Desiderio, i capolavori mancati rinominati da se stessi, orientaleggiando secondo le esigenze dello show moderno, Hong Kong Suzy e Shangai Lil (i nomi scelti dalla Signora erano Cupidigia e Bramosia), il sarto en travesti Grete Garbo (con vocale terminale - Grete- alla francese o alla svedese, come chiarisce lui), la fattucchiera «'a Monaca 'e tutte quante», parodia della sua omonima di Monza; la serva Sisina (nome non a caso baudeleriano, visto che i versi della lirica omonima verranno citati in conclusione da Desiderio), a cui si aggiungono i clienti lo Smilzo e Ciro, la $1^{\circ}$ e la $2^{\circ}$ vicina, la signora Compagnone, Giggino, il socio di malaffare della Signora e il bambino che funge da presenza-tramite tra realtà e teatro. Sullo sfondo il coro delle voci e Napoli a fare da platea.

Il prologo è chiaramente metateatrale: entrano in scena Giggino e il bambino, il primo si rivolge così all'altro:

\section{GIGGINO}

Guaglione mio, i debutti - qualunque debutto-sono fondamentali: importantissimi (p. 1$)^{18}$

Quindi gli indica la scena che prende vita e scompaiono. La consapevolezza metateatrale è d'altro canto uno dei fili rossi che attraversano tutto il testo. Già all'apertura della narrazione l'indicazione d'autore sulla prima battuta che spetta a Hong Kong Suzy è con tono molto teatrale, ribadito successivamente (con tono teatralissimo), quando lo stesso personaggio comincia a farsi il verso, per colpire la Signora:

HoNG Kong SuZY

Ah, Dio mio! Perché hai tagliato così asimmetricamente questo raso - il nostro corpo- se proprio non volevi fare di noi uno splendido tailleur di Chanel? (p. 10)

cessivi (il bianco, il rosso, l'oro), essa diviene null'altro che uno stimolo che conduce l'individuo verso aree della coscienza disgregate, malsane e dissociate, tendendo alla distruzione della persona e del contesto sociale, non riuscendo a produrre alfine l'oro nell'accezione, non volgare e materica, ma esoterica e intima (cfr. Marcello FuMAGALli, Dizionario di alchimia e di chimica farmaceutica antiquaria, Roma: Edizioni Mediterranee, 2000).

18. La numerazione delle pagine fa riferimento al copione vincitore dell'edizione 1985 del Premio Riccione. 
E in un crescendo continuo Hong Kong Suzy con gesto teatrale si butta ai piedi della Signora e dice, riferendosi a Desiderio:

Hong Kong Suzy [...] Oh Signora, Signora, pietà! Lasciateci vivere all'ombra di tale luce! Lasciateci respirare il suo profumo, il suo casto alito di eunuco. (p. 11)

D’altro canto Lui e Shangai Lil sono «il più puro estratto dei Quartieri Spagnoli» e fanno "scintille sui cartelloni», tanto da concedersi in "posa hollywoodiana».

A questo punto entra la cameriera Sisina che subito cede a un incanto da polpettone d'appendice:

SISINA

Uh, signò! Io m'ero incantata. Me pareva 'e sta' a triatre. Proprio una bella scena! 'A mamma a'na parte, $\mathrm{i}$ due figli a chell'ata... e in mezzo l'odio, l'odio nel divampare di una passione contrapposta, acerbosa (p. 14)

E la Signora si irrita e li caccia via dalla stanza:

Signora Jatevenne. Ascite. Commedianti (idem)

Inizia allora un lungo monologo in cui ci racconta com'è ascesa dal marciapiede del porto al ruolo di proprietaria di numerosi locali tra i Quartieri Spagnoli e Posillipo, dove fa esibire le sue creature, salvo essere interrotta da Giggino che entra giustamente applaudendo, come si fa a teatro.

Alla fine della pièce, quando tutto è consumato in mascheramenti e confusione delle lingue, la Signora così si rivolge a Desiderio, dopo la sua ribellione e mentre la sta canzonando assumendone i panni:

SIGNORA

...Ancora non ti sei stancato di rifarmi il verso. Prima hai giocato a me-giovane, adesso stai giocando a me... (p. 92)

E avendo Desiderio appena parlato in inglese, non è difficile leggere sotto al verbo giocare l'ambiguità semantica di "to play», traducibile sia come giocare che come recitare. Non si dimentichi, poi, che qui quasi tutti i personaggi agiscono travestiti, chi mutando sesso, chi condizione, chi bluffando. Il che si va a sommare ad una costante tentazione a trasfigurare le cose in altro da sé, a chiamarle sempre con altri nomi, a portare continue citazioni da avanspettacolo e soubrettismo da rotocalco, fino alla confusione identitaria finale tra la Signora e Desiderio (e qui il nome diventa più che mai parlante, nell'esprimere la forma dell'utopia), in cui tutto quello che è stato si vela dell'ombra della finzione. Ma ritorniamo alle preoccupazioni di forma che l'autore trasferisce direttamente alla Signora, in primis sotto l'aspetto della cura linguistica, tradita da 
continui svarioni, tanto da dare adito ad una vera e propria «questione della lingua»:

SIGNORA

SHANGAI LIL

SIGNORA
Lo sapete benissimo, che io esigo, che ho sempre esatto la forma (p. 3)

Sì la forma, la forma! E so' vint'anne che ce cantate'sta canzone! E noi, su questo, abbiamo sempre seguito i vostri dettami... seguito, come se dice?... seguito perfino pessi... pessiquamente

Pedissequamente (p. 3-4)

Corregge la Signora, e allora presto arriva il consiglio:

SHANGAI LIL

E parla napulitano! In questi casi, con lei, è sempre meglio a se fa capì più chiaro del possibile (p. 4)

Così poi, di fronte alla Signora reticente nel parlare in modo esplicito della prostituzione, tema che, per rinnegare il proprio passato, è diventato per lei tabù —e la competenza psicologica non è casuale - Hong Kong Suzy così la riprende, con altrettanta proprietà linguistica.

Hong Kong SuZY Oh, dite pure la parola. Non ci fa impressione. Ormai in questa casa, tra noi, è venuta meno ogni inibizione linguistica! (p. 6)

E poi:

SHANGAI LIL Il vostro difetto maggiore è che non apprezzate la sincerità: dire pane al pane e vino al vino (idem)

Affermazione che fa un po' da sintetizza tutta la storia. Allora subito arriva la ribattuta:

SIGNORA

Per quanto riguarda po' a chella cosa llà, che c'ê detto prima... a linguistica... bene: rispunneme nu poco... chi ha infranto per primo il patto? [...] Il nostro patto si poteva riassumere con tre verbe. Primo studiare, per diventare degli artisti. Secondo parlare solo italiano, in questa casa, per essere delle persone civili. E terzo, esibirsi, ad educazione compiuta. [...]. Per darvi un rango ho sudato, ho pagato... (p. 7)

Risposta:

Hong Kong SuZY Oh, non è che voi adoperiate il vocabolario Palazzi (p. 9) 
Il tema è talmente radicato che Desiderio, la sera del debutto è completamente smarrito per l'indigestione di parole e la scarsezza di realtà:

DESIDERIO

Ma le parole, le parole... le parole sono l'unico riferimento che io ho. La mia vita, fino a questo momento, è stata fatta solo di parole: libri, lezioni, precetti, spartiti, strofe... Tutte parole da mandare a memoria, con poca o nessuna esperienza concreta. (p. 51)

Lui, infatti, è uno dei pochi personaggi a non avere difficoltà nella gestione dell'italiano. Questi sono alcuni degli esempi di ricostruzioni ad orecchio in base all'esperienza, scambi suffissali, allosemie, difficoltà di analisi di tecnicismi, esotismi, cultismi o anche di semplici lessemi italiani che vengono distribuiti nel testo dagli altri personaggi: successo diventa successione (Giggino p. 2), contrastata: contrapposta (Sisina p. 14), Peluche D'Albania: Nanuccia, Peluccia, Parrucca, Perrucca (Sisina p. 18), volontariamente: volenterosamente (Sisina p. 19), sodalizio: solidarietà (Giggino p. 26), restia: restiva (Sisina p. 33), vincolanti: vincolabili (Sisina p. 41), ripugnante: ripugnevole (Sisina p. 42), sensuale: sessuale (Hong Kong Suzy p. 60), taumaturga: traumaturga (Sisina p. 78), in esclusiva: in esclusione (Sisina, p. 33), in esclusività (corregge la Signora, p. 33). E sono chiari esempi di italiano popolare rifatto, degni del grande Totò, a carico soprattutto della cameriera Sisina, che proseguono a livello morfologico e sintattico:

SISINA

Sì, sì: se l'invidia sarebbe una rogna, tutti quanti s'a stessero 'a grattà (p. 22)

SISINA [...] Il figlio suo carnale non le piaceva proprio. Assomigliava troppo all'americano'mbriaco, e la Signora è innamorata pazza della Bellezza. Poi, per secondo, l'ha dato ai zingari, per far scomparire le tracce di se stessa sulla scia dell'Americano (idem)

Diversi sono infatti i livelli linguistici gestiti dalle varie figure, anche se tutte sono caratterizzate dalla volontà di parlare meglio di quel che possono. Se ne possono distinguere almeno quattro: preteso italiano colto, italiano (sub)standard, napoletano e stretto gergo dialettale.

Sisina, portatrice di concretezza e, a modo suo, di verità, scesa a compromesso con la sceneggiata di casa solo per comodità, si rifa soprattutto alla saggezza dei modi di dire, contaminato da credenze magiche e superstiziose — di cui porterà la competenza nella storia - quando non televisive. Parla perciò preferibilmente in napoletano, con discese anche nel gergo più stretto:

SISINA

SHANGAI LIL

SISINA
Ccà ce vo' qualcheduno $c a$ 'o stupetèa!

Comm'ê ditto? Stu...

...Petèa! Stupetèa! Come a dire: incantesimo, malia 
Non ha vergogna ad esporre le sue difficoltà di comprensione del mondo falsificato e abbellito da griffes esterne:

$\begin{array}{ll}\text { SISINA } & {[\ldots] \text { Basta ca num me facite 'e sofisticate. Io nun sac- }} \\ & \begin{array}{l}\text { cio rispondere 'e domande complicate (a Shangai Lil } \\ \text { e Hong Kong Suzy, p. 20) }\end{array} \\ \text { GRETE GARBO } & \text { Comment? Avec les ciseaux! } \\ \text { SISINA } & \text { 'E che? } \\ \text { GRETE GARBO } & \text { Les ciseaux! Les ciseaux! 'E forbice! } \\ \text { SISINA } & \text { E vabbuò! Scusate. Perdonate la mia ignoranza... sape- } \\ & \text { te, io non sono mai stata all'estero... (p. 40) } \\ \text { SISINA } & \text { Tuttissimo, signò. Ce sta 'a tazza cu 'a pappa riale p'o } \\ & \text { Cigno... [...] i chiodi di garofano, le mandorle amare... } \\ & \text { e chest'ata rrobba ccà, ca nun saccio dicere (p. 15) }\end{array}$

E quindi il suo essere all'antitesi rispetto al mondo in vitro di Desiderio:
SISINA
DESIDERIO
[...] Ma non pensano a quello che dice la gente veden- doli salire le scale con chili duje mariuole?
SISINA

\section{Come?}
(aiutandosi con le mani) Mariuole! Ladri! Ladri di Bag- dad, capite? (p. 55)

Tanto che al suo uscire di scena così si congeda (e la confusione si esprime anche nella continua volubilità morfologica dal maschile al femminile, principale confusione interna alla casa, che si fonde nell'indistinzione vocalica postonica del napoletano):

SISINA

Bella casa, chesta! Bell $i$ facce d'assassine! Tutte quante! Tutte quante fingono di fingere tutto il giorno, da quando spunta'o sole fino a che tramonta (p. 88)

È Desiderio invece a portare l'italiano più depurato e talvolta letterario (per scelte lessicali e posizione dell'aggettivo):

DESIDERIO

Sono invidioso della loro leggerezza, delle loro episodi-
che, disattente letture, dei loro trucchi pesanti, della loro
naturale gaiezza... soprattutto sono invidioso, molto
invidioso della forza con cui affrontano la vita, quello
che c'è là, là fuori (p. 37 )

E, al contrario degli altri, non vira al disfemico, salvo quando imita la Signora, e al napoletano, neppure quando si alza la temperatura nervosa, anzi, dopo la serata di iniziazione, si esprime citando versi in lingua originale di Sylvia Plath, Jean Cocteau, Federico Garcia Lorca, Charles Baudelaire e decifra perfettamente il linguaggio magico della Monaca fattucchiera che tenta l'esorcismo: 


La Monaca
Desiderio
LA MONaCA
Desiderio
LA MONACA
Desiderio
...
LA MONACA

Sciarakkaddin?

Sì... sì, devo cacciarlo

Nabìk Ka ti kò?

Sì! Tra l'intestino e tutti gli sfinteri!

Flàm Ka ti kò?

(soffrendo) Molto. Sangue e acqua verde... qua (si tocca vagamente il ventre)

Legga bene colui che si contorce

Il collo non si storce,

bene mediti colui che si sacrifichi,

e i lembi suoi mortifica

si rettifichi l'ostinato

che già tanto ha crapulato... (p. 85)

La Monaca 'e tutte quante, che assieme a Grete Garbo, è il personaggio più comico della vicenda, è portatrice di un eloquio variegato, tra latino, napoletano, italiano e gerghi esoterici, il tutto per creare il mistero - ciarlatanoda maga di quartiere. Così esordisce in scena:

\section{La Monaca (a Sisina) Et ubi puella capta malo?}

Sisina, confusa, allarga le braccia

LA MONACA

(più sbrigativa) Addò sta’a figliola?

... Quella giovine... si avvicini. Che io le parli (idem)

Grete Garbo fa invece suo massimo vanto dell'essere stata a bottega da Cocò. Cocò Chanel, naturalmente, e allora francesizza, come si è già visto, per distinguersi dai provinciali, ma sfoggia anche citazioni colte:

GRETE GARBO

[...] Signora mia, rifletteteci bene: nolite mittere margaritas ante porcos. Questa’o ssapite meglio'e me, è una città di profani, di profanoni! (idem)

La massima escursione tra scurrile e sublime è contenuta nella lingua di Hong Kong Suzy e Shangai Lil che, se da un lato si fanno traduttori degli strani versi di Desiderio, dall'altro schiacciano apposta verso il basso la lingua civile loro impartita dalla Signora, intarsiandola soprattutto di segnali di mondo, naturalmente quello della cultura massmediatica, citata e riportata via lupanare:

Hong Kong Suzy Andiamo, ma andiamo. Ammettete che, in quanto ai nomi, siete antiquata nei gusti. Vuie nun conuscite'e regule dello show moderno

SHANGAI LIL

HONG KONG SUZY

...e ca un site 'na maitresse originale...

Dopotutto, Hong Kong Suzy e Shangai Lil fanno scintille sul cartellone. Ma pensate: il vostro locale si chiama Oriental, e noi arriviamo ballando una giava con gli occhi tirati così... 
SHANGAI LIL

Ma se proprio ci tenete posso pure cambiarlo il nome mio. Per me n'etichetta vale l'altra. Oppure mi ribattezzate voi, tenendo presente le moderne esigenze del mercato, si capisce. Che ne dite, per esempio, di SexyPagoda-Wolf? (p. 13-14)

La Signora, che porta in sé l'ascesa dal bordello al benessere, riesce, quasi sempre, a governare un italiano appreso tardivamente, soprattutto se è di fronte a Desiderio, salvo che però con l'accrescere dell'emotività è il napoletano la lingua delle sue viscere. Così si rivolge, parlando a singhiozzi e balbettii, al suo Angelo ribelle:

SIGNORA

...Nun è possibile! Nun è possibile! Tu me vuo' fa sule male! Tant'anne... tant'anne... ittate... scarpesate accussì... dint'a nu paro d'ore! Tant'anne sule pe' caccià fora na' puttana... 'na puttana peggio'e chill'ati dduje! Non è possibile... Tu si' asciuto pazzo, sì... tu me vuo' fa male, me vuo' fa murì... nun è possibile (p. 94)

Tra gli altri personaggi, citiamo ancora l'ulteriore nota di colore pluringuistico aggiunta da uno dei due clienti di Shangai Lil e Hong Kong Suzy, Lo Smilzo, mista tra napoletano e veneziano-spagnoleggiante:

Lo SMILZO

(riferendosi a Desiderio) Oh Johnny, però xè belo! Xè proprio una mona, me par! Mi me vodria cavà una voggia! (A Sisina) Uhè, camarera-caramba, xè veramente una dona, o xè solo un recion com'a leu? (p. 58)

All'orecchio, il risultato di un simile coacervo linguistico è quello di una partitura incentrata più sui significanti che sui significati, talvolta anche giocandovi e bisticciando a bell'apposta:

SISINA

HONG KONG SuZY

SHANGAI LIL
Ecco, ieri sera telefonò don Carmine Petruso, chilo... o menagèr, comme se dice?, il gestore di quel vostro locale, ma comme se chiama? Mannaggia, me scordo sempe o nome! (p. 17)

Del resto, l'avete detto pure voi: orfani e bastardi. Con una materia così, con una stoffa così, al massimo si può venire su come delle passabili coccottes

Credete forse che non ci scotti, che non ci bruci il rimorso di avervi deluso? (p. 10)

Vengono in mente i vecchi repertori della Commedia all'improvviso, se non fosse che qui il cortocircuito si fa ancora più esplosivo, poiché è innescato in un'atmosfera di fondo nero, dove il riso, quando c’è, è amaro.

Il piano stilistico al quale si può ascrivere la maggior parte del teatro di Moscato è, infatti, quello tragico: spesso letali sono gli esiti delle vicende rap- 
presentate ed è indubbio che, a caratterizzare la natura tragica, concorre un elemento di irreparabilità che quasi sempre sfocia nella catastrofe. Ma soprattutto, a radicare nel terreno tragico la materia del drammaturgo napoletano, vale la caratterizzazione di tutto il suo teatro, e cioè quella lacerazione fra due tensioni opposte che non si ricompongono mai, nella quale, appunto, viene individuato il nucleo essenziale della tragedia.

Non si tratta tuttavia di un tragico, per così dire, puro, perché esso sempre si unisce alla dimensione comica e a quella lirica, secondo una predilezione per le mescolanze stilistiche e linguistiche che caratterizza tutta la modernità e, come si è visto, è cifra peculiare del suo stile. Ma bisogna precisare che il comico di Moscato, anche quando mostra di avere alle spalle la tradizione più consolidata dell'avanspettacolo e della farsa napoletana, non è disteso né facile: assume piuttosto forme ironiche, sarcastiche, aggressive; spesso è adottato come elemento di rassicurazione contro i fantasmi della morte e l'ossessione della sessualità, i due aspetti tematici forse più significativi e più persistenti della sua opera.

E passiamo, dunque, proprio ad individuare le ossessioni tematiche, perché di questo si tratta, del testo. Le preoccupazioni di forma cui si è già fatto cenno, si sviluppano nel testo in una delirante presenza di forme precipitate in pupaz$z i$, manichini, bambole - appellativi con cui vengono reificati i rapporti umani e soprattutto l'allevamento di angeli della Signora-, modelli di moda, griffes di stilisti, nomi-etichetta, per raggiungere il culmine negli uccelli impagliati dentro alla gabbia che fanno da specchio, da forma, a tutta la vicenda. E infatti anche il mondo degli uccelli ha tutto un proprio corrispettivo nomenclatorio che va dalle canarie, alle tortore-angeli imperfetti Hong Kong Suzy e Shangai Lil, rispetto al Cigno-Desiderio che quando parla cinguetta, perché lui sì è l'Angelo della Misericordia. E così dal tema degli uccelli si passa a quello del sacro e a tutti i suoi simboli. Dai modi di dire di Sisina, della Signora e di Hong Kong Suzy e Shangai Lil: Giesù-siccame-'a-lengua, Tu vuo' trasì troppo dint'a scazzotta d'o sacrestano, Tutto a Gesù (Desiderio) e niente a Maria, alle Maronne di cera, alle immacolate spoglie dell'Angelo, ovvero sempre Desiderio: il Cherubino, la Casta guerriera d'Orleans, atteso prima del rito di iniziazione all'Etoile $d u$ Nord, cioè la Stella, unico altare — chiamato anche tempio — in cui può esibirsi la Casta Diva (per via meta-teatrale), d'a processione,'o Sacramento, per non parlare dell'intervento della Monaca'a tutte quante, con cui però presto si vira dal sacro al mondo para-spirituale ed esoterico partenopeo. E allora ecco tutti gli apparati e gli operatori della magia, della cartomanzia, le fattucchiere e le formule contro il malocchio: stanotte, premunitevi contro il malocchio! Mettete un paio di forbici aperte sotto il letto, metteteci nu spicchio d'aglio mmiez'a mano, scupatele appriesse, vuttate nu poco 'e sale pe 'e scale... (p. 49) 
Raccomanda il sarto alla Signora prima dell'uscita di Desiderio per l'Etoile.

$\mathrm{Ma}$ a lato della magia popolare, più o meno rimpastata nei codici dei cialtroni moderni, c'è tutta la chimica fai-da-te — l'alchimia — della Signora fatta di premolina, olio di azulene, ormonal, balsamo di mango, nembuthal, barbiturici e chi più ne ha più ne metta, per ottenere l'androgino perfetto. Prima di rilanciare l'ipotesi di una possibile, conseguente, lettura alchemica sottotraccia, si noti come la struttura ossimorica di Moscato, che punta a ricongiungere e a far combaciare specularmente ciò che è alto e ciò che è basso, verificabile anche nei temi riportati, sia riconducibile in fondo alla matrice che unisce l'altare e il lupanare, a cui tutta la sacralità metaforicamente fa riferimento. Anche in questo senso il percorso di Desiderio, stando anche alle dichiarazioni di poetica di Moscato, è leggibile come un percorso iniziatico.

E ritorniamo ora al significato alchemico celato nel titolo. La prima fase dell'Opus è, appunto, la nigredo, l'Opera al nero, un cammino di morte e rinascita per raggiungere il secondo stadio, quello dell'albedo. Durante questo passaggio viene a comporsi il rebis, la res bina, denominazione data dagli alchimisti alla figura simbolica che si produce dalla prima decozione dello spirito minerale mescolato al suo corpo, "poiché è fatto da due cose, cioè dal maschio e dalla femmina, ovvero dal solvente e dal corpo solubile». ${ }^{19}$ E secondo la Tabula Smaragdina (di Ermete Trismegisto) il rebis, generato dal Sole e dalla Luna, raccoglie in sé le virtù essenzialmente unite, ma esteriormente polarizzate, del Cielo e della Terra, presenti nel testo, come si è visto, nel continuo rimando del lupanare al mondo sacro. Il Rebis evoca l'uovo filosofico degli alchimisti, la cui separazione in due parti corrisponde alla manifestazione per polarizzazione dell'unità primaria. Il suo germe è infatti una figura androgina: l'androgino perfetto degli alchimisti, in cui sono fusi animus e anima. Questo androgino è dunque una figura sacra, immortale alla vera vita, a cui non è difficile sovrapporre l'ambizione suprema della Signora, incarnata da Desiderio, in qualche modo anche figura cristica, così espressa da Hong Kong Suzy:

Hong Kong SuZY ... Voi siete una monomaniaca, una fissata! Non vi bastano le bambole, i femminelli, i travestiti perfetti! Voi volete pure i Santi, 'e Maronne, gli Angeli! (p. 75)

E il percorso di morte-nigredo di Desiderio passa attraverso l'iniziazione nel bordello-tempio — sotto cui si cela la figura del palcoscenico-athanator (alambicco alchemico) e dunque del teatro come lavacro spirituale- eretto solo per lui dalla Signora con il nome, eloquente, di Etoile du Nord. Il rituale di preparazione, vestizione, attesa estatica della processione corale all'esterno della casa, fanno da contorno al battesimo della vita, la vera vita, da lui tanto attesa durante il periodo di reclusione. Al suo ritorno dal tempio, Desiderio non è più lui, parla una lingua strana, che confonde e mescola insieme tutti

19. Jean-Alain Chevalier-Gheerabrant, Il Dizionario dei simboli, Milano: Rizzoli, 1986, p. 283. 
gli idiomi e si scandisce in versi, proprio come l'alchemico sacro gergo degli uccelli (!). Al suo risveglio appare un alba-albedo nuovissima che lo contagia con il proprio biancore:

DESIDERIO

Dies Angeli. L'aurora è ai vetri. Porta male salutarla con dispetto...

Dies Angeli. È proprio un'aurora speciale: candida, bianchissima. Presto anche i miei capelli saranno candidi così... (p. 91)

A questo punto il filosofo che ha intrapreso il cammino dell'opus, divenuto "fanciullo regale», non è più sottoposto all'esistenza del corpo, essendosi questo congiunto con l'anima nella vita cosmica.

Sempre secondo la scrittura alchemica, nel processo di morte-nigredo la prostituita di Babilonia — che rappresenta questa fase dell'Opera al neroviene santificata nella Vergine dei Filosofi, processo che non può mantenersi in questo mondo, e ancora qui nel testo si segue il tema:

LA MONACA

Questa casa? Tenete 'na bella facciatosta a chiamarla casa! Io aggio capito tutte cose, tutto! Questa è una bolgia dantesca, sì, quest'è 'na Babbilonia! Una Babbilonia!

(alla Signora) Visto che impedisci, peccatrice, la mia santa opera di guarigione, tieni a mente 'sti parole, tienile buone a mente tu: tu... tu sei in extremis... (p. 87)

Ma il processo di trasfusione alchemica non va a buon fine, aprendo dunque il varco alla tragedia. Lo stesso Desiderio, da tutti definito filosofo (Hong Kong Suzy e Shangaj Lil ricordano che ha letto anche Il libro tibetano dei morti, un classico della religiosità orientale sul destino dell'uomo dopo la morte) così rimprovera in conclusione alla Signora le sue odiose alchimie:

Desiderio

E finitela una buona volta! Basta con le vostre ridicole manie! Ma quale Angelo, quale? L'Angelo! Gli angeli non si costruiscono con l'ormonal, coi siliconi, coi trucchi, coi preparati all'azulene... con gli specchi! Gli Angeli! Le vostre sono sbobbe con cui si divertono solo le più miserabili checche (p. 94-95)

Non c'è più alternativa: il processo di transustanziazione non può proseguire qui e Desiderio verrà ucciso dalla Signora come in passato aveva fatto con tutti gli Angeli abortiti. Così le spiega in breve la vita Hong Kong Suzy: puntone 'e vico v'aspetta... pe' s'annettà 'e mane sporche ncuoll'a vvuje (p. 75) 
Ma, nonostante il finale nero, Moscato ci indica anche un'altra importante istanza del moderno, ovvero che l'unica modalità di manifestazione possibile oggi della tragedia è la sua contaminazione comica, o meglio la sua carnevalizzazione: ${ }^{20}$

SHANGAI LIL

Eh già! È proprio una gran bella mascherata, Madame. Un grande, folle, eccezionale Carnevale! (p. 80)

E proprio in questo carnevale in cui ogni cosa è anche "figura», o meglio maschera di qualcos'altro, non si può non intravedere sotto gli intrugli e gli alambicchi della Signora una contro-figura della funzione dell'autore. La fusione alchemica di polarità opposte è infatti anche la base della scrittura di Moscato; il risultato non può dunque essere se non un teatro che è, artaudianamente, anche un cammino di conoscenza di sé, il quale si riversa sul lettore-spettatore attraverso un processo analogo di interiorizzazione, che potremo definire di catarsi post-aristotelica.

Siamo di fronte ad un testo-sistema in cui tout-se-tient: la microstruttura e la macrostruttura si corrispondono in ogni parte. E in questo configurarsi alchemico dell'opera come oggetto frattale, che si seziona in porzioni sempre formalmente identiche secondo un'unica legge che le fa convergere tutte ad un'unica origine, anche se strutturalmente ab-normi, barocche appunto, sta la tenuta del teatro di Moscato. La possibilità del dramma scaturisce dunque in Moscato dal cozzo tra materia e spirito, ovvero dall'imperfettibilità della prima nel tendere verso il secondo e dallo scacco che ne consegue; funzione del teatro diventa perciò la rivelazione, sotto aspetti anche linguisticamente tollerabili, di nuclei di essere (l'apollineo e il dionisiaco nietszchiani), per cui la finzione è coagulo delle forme via via assunte da questa rivelazione secondo il principio alchemico del solve et coagula. La sua scrittura teatrale $\grave{e}$, infine, scrittura simbolica, in cui la fictio metateatrale è funzionale alla conoscenza che si libera dai simboli teatrali stessi e dalle loro opposizionicongiunzioni.

Portando il discorso ad un ambito più generale, si può anzi avanzare l'ipotesi che questa duplicità di livelli, per cui la superficie testuale e il sottotesto si implicano a vicenda ma conducono, l'una, al semplice svolgimento di una storia, l'altro, ad un percorso più generale che va oltre la storia contingente sia proprio consustanziale alla scrittura teatrale tout court. In questo senso possiamo dire che non solo la vocazione della scrittura teatrale italiana sia antimimetica e trovi i suoi migliori risultati in alcuni inventori di lingua, ma che sia la lingua stessa del teatro a dover essere per sua natura mascherata, riferen-

20. Si fa riferimento naturalmente a Michail BACHTIN, Dostoevskji, Torino: Einaudi 1968, p. 140178 e ID., L'opera di Rabelais e la cultura popolare, Torino: Einaudi, 1979. Invece per suggestioni tra teatro e alchimia, teatro e mistica cfr. Umberto ARTIOLI e Francesco BARTOLI, Teatro e corpo glorioso. Saggio su Antonin Artaud, Milano: Feltrinelli, 1978 e Umberto ARTIOLI, L'officina segreta di Pirandello, Bari: Laterza, 1989. 
dosi sempre anche ad ambiti di senso più profondi, ovvero raccogliendo dentro alla struttura superficiale una ben più densa struttura profonda.

Il teatro solo nel Naturalismo si pose il proposito di rispecchiare la realtà (ma ancora con molti residui mitologici che resero possibile l'operazione): la sua natura è quella di essere programmaticamente finto, o meglio di intrattenere con la realtà lo stesso rapporto che c'è tra il sogno e la veglia, per cui la finzione diventa forma, attraverso una propria peculiare grammatica, di una realtà più vera. Perciò la lingua del teatro diventa la maschera con cui è possibile accedere ad alcuni - pochi, ripetitivi, quasi tutti già presenti negli archetipi della tragedia classica - nuclei di verità folgorante che se non trovassero quel codice non sarebbero comunicabili. Il teatro è il luogo il cui contatto provoca uno $c h o c$, la rottura momentanea di un confine, la rivelazione istantanea dalla bocca della scena di un segreto impronunciabile. Teatro è vedere ciò che si deve nascondere: theatron e skenè, perché la maschera cela nelle proprie cavità le paure umane, l'angoscia, i mostri, i terrori; rende visibile tutto ciò che sfugge al controllo dell'uomo per provare ad esorcizzarlo. Dove non conta la coerenza alle leggi naturali ma la coerenza alle proprie leggi. Lo stesso possiamo dire, in conclusione, della sua lingua: si tratta di una lingua finta perché verissima. 